Central Washington University

ScholarWorks@CWU

All Faculty Scholarship for the College of the Sciences

$1-2002$

Atmospheric turbulence conditions leading to focused and folded sonic boom wave fronts

Andrew A. Piacsek

Follow this and additional works at: https://digitalcommons.cwu.edu/cotsfac

Part of the Aerodynamics and Fluid Mechanics Commons, Atmospheric Sciences Commons, and the Physics Commons 


\title{
Atmospheric turbulence conditions leading to focused and folded sonic boom wave fronts
}

\author{
Andrew A. Piacsek ${ }^{\mathrm{a})}$ \\ Department of Physics, Central Washington University, Ellensburg, Washington 98926
}

(Received 12 July 2000; accepted for publication 9 April 2001)

\begin{abstract}
The propagation and subsequent distortion of sonic booms with rippled wave fronts are investigated theoretically using a nonlinear time-domain finite-difference scheme. This work seeks to validate the rippled wave front approach as a method for explaining the significant effects of turbulence on sonic booms [A. S. Pierce and D. J. Maglieri, J. Acoust. Soc. Am. 51, 702-721 (1971)]. A very simple description of turbulence is employed in which velocity perturbations within a shallow layer of the atmosphere form strings of vortices characterized by their size and speed. Passage of a steady-state plane shock front through such a vortex layer produces a periodically rippled wave front which, for the purposes of the present investigation, serves as the initial condition for a finite-difference propagation scheme. Results show that shock strength and ripple curvature determine whether ensuing propagation leads to wave front folding. High resolution images of the computed full wave field provide insights into the spiked and rounded features seen in sonic booms that have propagated through turbulence. (C) 2002 Acoustical Society of America.
\end{abstract}

[DOI: $10.1121 / 1.1377631]$

PACS numbers: 43.28.Mw, 43.25.Cb, 43.50.Vt [MRS]

\section{INTRODUCTION}

The stochastic nature of turbulence precludes a complete analytical solution to the problem of predicting how a sonic boom will distort after passing through the atmosphere's turbulent boundary layer (TBL). In order to predict the precise wave form observed at the ground, it would be necessary to specify the velocity, temperature, and density of the atmosphere within a considerable volume. The best we can hope for, then, is to predict the average effects of turbulence on certain broadly defined features of the wave form, and to compute the probability of certain extreme distortions, such as spikes, being present.

Studies of human response to sonic booms suggest that three main factors contribute to annoyance: peak amplitude, rise time, and overall spectral content. ${ }^{1}$ Although they are related, there is some degree of independence among these factors. Clearly, a model for sonic boom propagation through turbulence should predict average values for these wave form characteristics as a function of general turbulence parameters. However, signatures of sonic booms created during test flights show considerable variability: Both peaked and rounded wave forms have been observed at different microphones during the same flight, and among different flights at the same microphone. ${ }^{2}$ This suggests that a sonic boom distortion model should also predict probabilities of certain extreme wave forms.

Two distinct modeling approaches have received significant attention: Crow's scattering theory ${ }^{3}$ and Pierce's model of wave front rippling leading to focusing and folding. ${ }^{4,5}$ The main result of the first-order scattering theory is a power law dependence of rms pressure fluctuations on the distance of the observer behind the shock front (also expressed as time

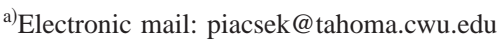

after shock arrival). This power law is in approximate agreement with experimental observations, ${ }^{6}$ at least for times significantly later than the shock arrival. An extension by Plotkin and George to incorporate second-order scattering perturbations permitted a calculation of shock thickening ${ }^{7}$ and a reformulation by Plotkin ${ }^{8}$ yielded a calculation of the spectral content.

The scattering theory necessarily yields a statistical result, stemming from a statistical description of turbulence; it does not give a reliable prediction of a particular outcome for the fine-scale features of a sonic boom signature. It is not clear, then, whether this theory can give a satisfactory prediction of the probabilities of particular wave form shapes. Moreover, molecular relaxation and nonlinearity are incorporated into the first-order (linear) scattering theory in an ad hoc manner, leaving open the question of the significance of these effects on the mechanism of scattering.

This paper describes an effort to apply a numerical propagation scheme to Pierce's theory of rippled wave fronts. In this model, the first-order effect of turbulence is to refract the incoming steady-state sonic boom, producing a rippled wave front. Where the ripple is concave in the direction of propagation, the shock will focus and possibly form folded wave fronts and caustics. The pressure behind the shock (near the axis of focusing) is strongly influenced by diffracted waves originating along the wave front. Where the ripple is convex, geometric spreading leads to decreased amplitudes. Pierce showed that where focusing occurs, the shock front becomes spiked, and where defocusing occurs, the shock front becomes rounded. ${ }^{4}$

Wave front ripples will have many length scales, corresponding to the inertial subrange of turbulence scales. The cumulative effect of focusing and defocusing at many scales could conceivably produce a thickened shock front, as well as either a net peaked or rounded wave form with smaller 
spikes superimposed. Pierce combined this approach with Crow's statistical model of turbulence and derived a probability density function for the arrival time of microshocks produced by focusing. ${ }^{5}$ The staggered arrival of microshocks had the effect of thickening the overall shock. This analytical approach, while initially encouraging, rested on many assumptions and is somewhat difficult to interpret.

Since that time, computer speed and availability has reached the point where it is now the standard approach to numerically solve the equations suggested by these models of sonic boom propagation. For Pierce's model to yield a statistical prediction of sonic boom distortion, an initially plane wave front must be propagated through many realizations of turbulence, as in a Monte Carlo method, and statistics compiled from the many outcomes. One advantage of this "brute force" approach is that particular model outcomes may bear some resemblance to actual outcomes, unlike a solution that incorporates an ensemble average of turbulence effects. In this way, probabilities of particular outcomes (extremely peaked or rounded wave forms, for example) may be computed.

As a step in this direction, the present paper discusses numerical results obtained by propagating the positive phase of a sonic boom with singly and multiply rippled wave fronts. Turbulence is not incorporated directly into the propagation model, but serves only to produce the initial rippling. This simplification permits the study of wave form distortion as a function of propagation distance from a particular realization of wave front rippling. It also makes possible an interpretation of the role played by different rippling scales with regard to the location and magnitude of spikes or other identifiable features of the distorted wave form. Another advantage of assuming that the medium is inhomogeneous only within a single thin layer is the ability to assess the time scales of the evolution of certain features of wave form distortion, such as primary and secondary spikes. Results presented here suggest that some of these time scales are comparable to the propagation time through the entire atmospheric boundary layer; thus, it may be that distortions observed at the ground are, to first order, due to wave front rippling that occurs near the top of the boundary layer, since subsequent rippling may not have had sufficient time to develop features associated with focusing.

Section I describes the numerical experiment. A modified version of the NPE program by McDonald and Kuperman ${ }^{9}$ is used to propagate the positive phase of a sonic boom-like $N$ wave whose wave front is rippled. The precise form of the rippling is related to a simplified description of turbulence. As discussed previously, no turbulence is incorporated into the propagation model beyond the initial rippling.

A detailed discussion of the focusing behavior of a weak shock with a finite rise time is presented in Sec. II. This builds on previous work by the author ${ }^{10}$ which examines the propagation of a step shock with a single concave ripple in the wave front. It is shown that two parameters, one associated with wave front curvature and the other with shock overpressure, govern whether geometric propagation predominates, such that wave front folding occurs beyond the focal point, or nonlinear effects predominate, causing the concave wave front to straighten. The range of parameter values corresponding to shock amplitudes and ripple scales typical of sonic booms entering the TBL is shown to encompass the transition between folding and shock dynamic behavior.

Numerical results for singly and multiply rippled wave fronts are presented in Sec. III. Different ripple dimensions and different observer locations are explored for singly rippled wave fronts. Finally, the case of a sonic boom wave front containing two ripple components is examined. Depending on the observer location, the multiply rippled wave front produces either extra spikes or a delayed onset of the shock peak.

\section{NUMERICAL MODEL}

\section{A. Overview}

A modified form of the NPE program developed by McDonald et al..$^{9,11}$ is applied to propagation of sonic booms with slightly curved wave fronts. The NPE is a time-domain approach that models first-order nonlinear wave propagation that may be diffracted at small angles from the primary axis. The linearized version of the NPE is essentially the timedomain equivalent of the parabolic equation often applied to sound beams. The frame of reference is an observer moving along the primary axis with the ambient sound speed, $c_{0}$; this eases constraints on the grid spacing and time step size needed for computational stability. Other features of the algorithm make it robust at handling steep gradients in the solution.

The NPE was developed primarily to model shock propagation underwater. To make the program suitable for sonic boom propagation, the effects of thermoviscous dissipation and molecular relaxation have been incorporated. With the primary direction of propagation coinciding with the $x$ axis and assuming diffraction occurs only in the $x-y$ plane, our modified two-dimensional NPE can be written as follows:

$$
\frac{\partial p}{\partial t}+\left(c_{0}+\beta \frac{p}{\rho c_{0}}\right) \frac{\partial p}{\partial x}+\frac{c_{0}}{2} \int_{\infty}^{x} \frac{\partial^{2} p}{\partial y^{2}} d x^{\prime}-\delta_{\mathrm{eff}} \frac{\partial^{2} p}{\partial x^{2}}=0,
$$

where $p$ represents acoustic pressure, $c_{0}$ the ambient sound speed, $\beta$ the parameter of nonlinearity, and $\delta_{\text {eff }}$ an effective dissipation coefficient that incorporates thermoviscous damping as well as the effect of molecular relaxation for a steady state shock.

It should be noted that this method of accounting for molecular relaxation is not strictly valid for a focusing shock, since focusing is not a steady-state process. Moreover, near focal points and caustics peak pressures may exceed $150 \mathrm{~Pa}$, at which point vibrational energy states appear frozen to the passing shock front and no longer contribute to dispersive shock thickening. Modeling the effects of relaxation will be discussed in more detail in the following section.

A two-dimensional acoustic field, $p_{0}(x, y)$, is specified as the initial condition on the discretized coordinates $\left(x_{i}, y_{j}\right)$. The algorithm then marches in time, solving a finite difference approximation of Eq. (1). At specified times, the solu- 
a)

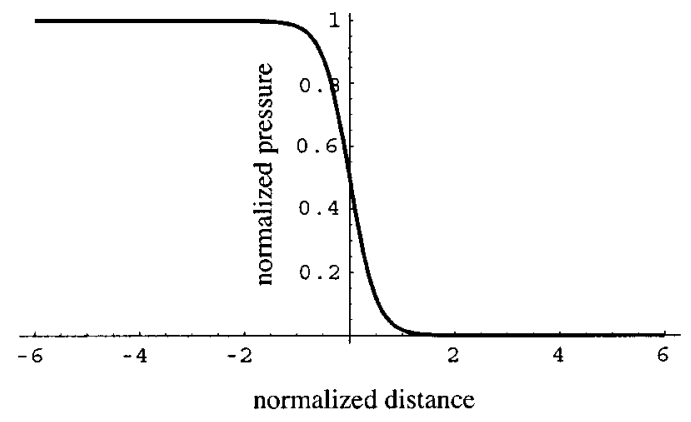

b)

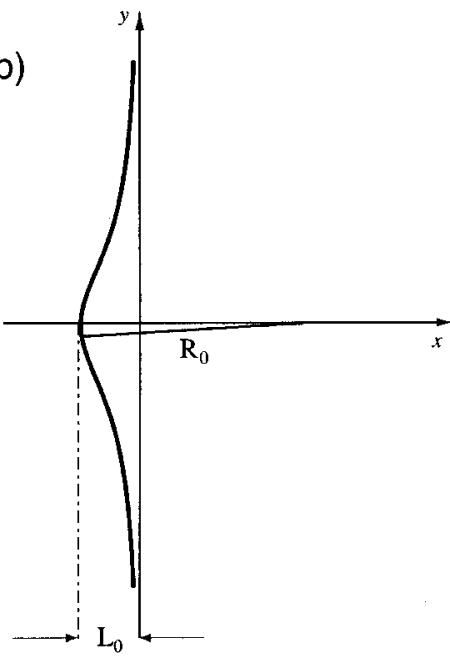

FIG. 1. (a) Wave front with parabolic ripple. $R_{0}$ is the radius of curvature at $y=0 ; L_{0}$ is the ripple amplitude. (b) Hyperbolic tangent profile of step shock. Distance along the $x$ axis is normalized with respect to the shock width; pressure is normalized with respect to the shock amplitude. tion array is written to disk, then rendered as a threedimensional image. The high spatial resolution makes it possible to visualize the entire wave field as a smooth surface, in which the details of the shock front may be discerned.

The computational boundaries parallel to the $x$ axis act as rigid, frictionless walls, reflecting incident waves without attenuation or phase shift. This type of boundary was chosen for its simplicity and reliability. However, with such simple boundaries, care must be taken to make the domain sufficiently large that, within propagation times of interest, reflected waves do not contaminate the solution near the shock front. It is also necessary to ensure that the initial wave front is perpendicular to these boundaries.

The acoustic pressure is set to zero everywhere ahead of the shock front. A grid tracking algorithm prevents the shock front from advancing too close to the front of the computational domain.

Computations were performed on a DEC Alpha workstation, with a typical grid size of 700 by 1000 points. With uniform grid spacing, the number of grid points needed to be large in order to achieve satisfactory resolution of the shock front while encompassing the entire positive phase of an $N$ wave.

\section{B. Initial conditions}

The initial pressure field consists of the positive phase of an $N$ wave that has a steady-state shock profile and a slightly curved wave front. In the vicinity of the shock, the wave form is described by a hyperbolic tangent function [shown in Fig. 1(a)]; behind the shock front, the wave form amplitude decreases linearly to zero. The hyperbolic tangent shock profile corresponds to the steady-state solution of Eq. (1) obtained when the initial condition is a planar step function. Traversing the $x$ axis, the shock is specified by

$$
p_{0}(x)=\frac{P_{\mathrm{sh}}}{2}\left(1-\tanh \frac{2 x}{l_{\mathrm{sh}}}\right),
$$

where $P_{\text {sh }}$ denotes the peak shock pressure and $l_{\text {sh }}$ is the Taylor length (which can be thought of as the shock width), given by

$$
l_{\mathrm{sh}}=\frac{8 \delta \rho_{0} c_{0}}{\beta P_{\mathrm{sh}}} .
$$

When only thermoviscous effects are considered, $\delta$ $=\delta_{\mathrm{cl}}=1.86 \times 10^{-5} \mathrm{~m}^{2} / \mathrm{s}$ is the classical damping coefficient in air. In steady-state conditions, with the shock overpressure between $30 \mathrm{~Pa}$ and $120 \mathrm{~Pa}$, the early portion of the shock rise is dominated by dispersion associated with $\mathrm{O}_{2}$ relaxation. ${ }^{12}$ The fully dispersed shock front has a rise profile that is similar to the hyperbolic tangent form produced by classical (thermoviscous) damping. The effects of $\mathrm{O}_{2}$ relaxation can thus be modeled by replacing the classical dissipation coefficient with an effective dissipation coefficient, $\delta_{\text {eff }}{ }^{13}$

$$
\delta_{\text {eff }}=\delta_{\mathrm{cl}}+c_{\mathrm{fr}} \tau_{\Delta C}=1.67 \times 10^{-3} \mathrm{~m}^{2} / \mathrm{s},
$$

where $\tau$ is the relaxation time of $\mathrm{O}_{2}, c_{\mathrm{fr}}$ is the frozen shock speed, and $\Delta c$ is the difference between the frozen and equilibrium shock speeds. The frozen shock speed corresponds to a shock rise time that is much shorter than the molecular relaxation time (such that molecular motion appears frozen to the shock), whereas the equilibrium sound speed corresponds to a shock with a sufficiently long rise time that molecular vibration states are always in equilibrium throughout the passage of the shock. Note that, in the limit of large shock amplitude, $\Delta c$ approaches zero and $\delta_{\text {eff }}$ approaches $\delta_{c l}$.

The wave front [depicted in Fig. 1(b)] lies nominally in the $y-z$ plane at $x=0$. The shallow ripple is specified as a variation along the $y$ axis of the shock arrival time, $\tau_{0}(y)$, at $x=0$. An example of the form of $\tau_{0}(y)$, used in the case of a step shock with a single concave ripple, is

$$
\tau_{0}(y)=\frac{L_{0}}{c_{0}}\left[1+\frac{L_{0}}{2 R_{0}}\left(\frac{y}{L_{0}}\right)^{2}\right]^{-1},
$$

where $L_{0}$ is the maximum depth of the ripple and $R_{0}$ is the minimum radius of curvature of the wave front; both occur at $y=0$. Note that the ripple is symmetric about $y=0$; thus, the $x$ axis shall be referred to as the "central axis." At large values of $y, \tau_{0}$ approaches zero, so the wave front is nominally planar except in the vicinity of the central axis. Wave front curvature exists solely in the $x-y$ plane; the wave front is uniform along the $z$ axis [normal to the page in Fig. 1(b)]. 
The geometry of focusing is then two dimensional.

It is convenient and instructive to define nondimensional parameters associated with this initial shock profile and wave front curvature. The shock thickness is described by the parameter $\mathcal{T}=l_{\mathrm{sh}} / L_{0}$, the wave front curvature is described by $\mathcal{C}=L_{0} / R_{0}$, and the shock amplitude is characterized by $\mathcal{P}$ $=\beta P_{\mathrm{sh}}\left(\rho_{0} c_{0}^{2}\right)^{-1}$. Each of these parameters corresponds to a physical process that plays some role during wave front focusing.

The shock thickness parameter, $\mathcal{T}$, can be associated with diffraction that occurs within the shock front, referred to here as "inner diffraction." When $\mathcal{T}$ is zero, the shock front is perfectly abrupt and, in the absence of nonlinearity, propagates exactly according to geometric theory. This leads to a singularity in acoustic intensity at the point of first focus, $(x, y)=\left(R_{0}, 0\right)$, where rays launched from the immediate vicinity of $y=0$ intersect. However, a nonzero value for $\mathcal{T}$ ensures that the shock amplitude will remain finite, even at the focal point, since frequencies comprising the shock front are not arbitrarily high and will diffract away from the wave front normal when the length scale of wave front curvature is comparable to that of the shock thickness (where $\mathcal{T} \approx 1$ ). Inner diffraction will always occur within a region arbitrarily near the focal point as long as $\mathcal{T}$ is nonzero.

The wave front curvature parameter, $\mathcal{C}$, indicates the amount of "outer diffraction" from points along the curved wave front. Diffracted waves originating from beyond the inflection points of the initial wave front are responsible for the familiar logarithmic amplitude profile behind the shock front at caustics. ${ }^{14} \mathcal{C}$ is inversely proportional to the time required for the shock to reach the focal point; it is directly proportional to the magnitude of diffraction effects behind the shock within a unit distance (or time) of propagation.

The shock strength parameter, $\mathcal{P}$, is directly proportional to the strength of nonlinear effects, such as steepening. This parameter can also be expressed in terms of the mach speed of the shock front, $M=v_{\mathrm{sh}} / c_{0}=1+0.5 \mathcal{P}$.

These three parameters are completely independent of each other. Each represents the degree to which the corresponding physical effect governs the shock front evolution at $t=0$. By constructing several initial shock fronts that differ in the relative sizes of these parameters, it is possible to assess the relative importance of each physical process (inner diffraction, outer diffraction, and nonlinearity) upon the shock profile evolution near a focus.

It should be noted that these parameters are defined for the initial state, only. They are useful, nonetheless, because propagation in a homogeneous medium is determined by the initial state. The curvature parameter, $\mathcal{C}$, does not completely specify the initial wave front, but it does correspond to the rate at which diffraction effects contribute to the solution along the central axis.

The following briefly describes some numerical results showing that $\mathcal{P}$ and $\mathcal{C}$ govern whether a step shock with parabolic wave front curvature will propagate according to geometric acoustics or shock dynamics theory.

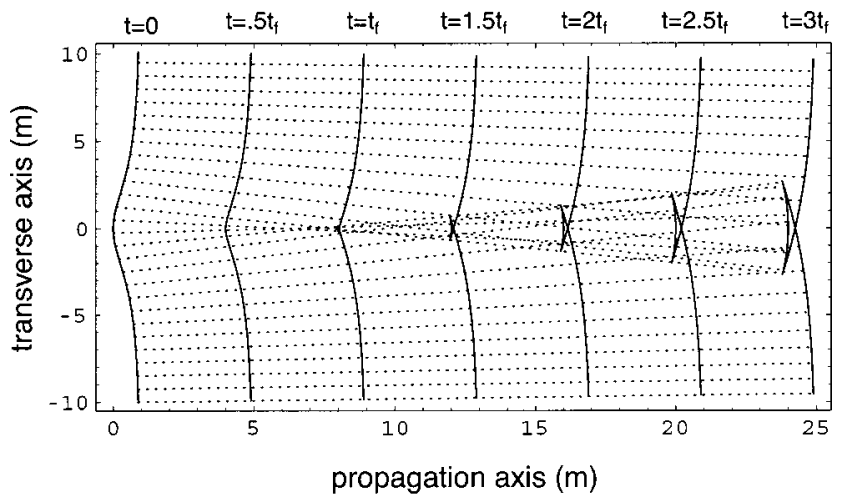

FIG. 2. Geometric evolution of initially concave shock front. Shown is a progressive sequence of wave fronts (solid lines) drawn at equal time intervals. The dashed lines are the ray trajectories.

\section{Step shock with a single focus}

Numerical experiments investigating the evolution of shock profiles in the region of a geometrical focus were carried out to examine the relative importance of diffraction and nonlinearity in the behavior of the shock front. The wave front is curved as described in Sec. II B, illustrated in Fig. 1(b), and the initial shock profile is the hyperbolic tangent function given by Eq. (2), shown in Fig. 1(a). Behind the shock front, the pressure amplitude is constant. Henceforth, this initial condition will be referred to as a "step shock," with the understanding that the shock thickness is finite.

The step shock, rather than an $N$ wave, was chosen for this preliminary investigation in order to have a computational array that was no larger than necessary to observe the evolution of the shock itself and the vicinity immediately behind it. Within this region, the slowly decreasing pressure field of a sonic boom-like $N$ wave is nearly indistinguishable from a constant pressure field.

The numerical results for the initial condition just described are compared to the linear geometrical evolution of a discontinuous shock that has the same wave front curvature. Figure 2 depicts the geometrical propagation of the rippled shock; the solid curves are the wave front at successive time intervals, and the dashed lines trace rays that leave from the initial wave front on the left. After the point where rays first intersect $\left(t=t_{f}\right)$, the wave front becomes folded, forming the fish tail, or delta, pattern characteristic of this focusing geometry. Caustics are located at the extremes of the fish tail, $y_{c}(x)$, where the wave front folds back on itself. An observer located within these extremes $\left(-y_{c}(x)<y<y_{c}(x)\right.$ at a distance $x$ from the initial wavefront) experiences three shock fronts passing by, except at $y=0$, where two shocks are observed; outside this region only one shock is observed.

It is expected that nonlinear effects may prevent, or alter, the geometrical propagation shown in Fig. 2 because shock dynamic theory predicts self refraction of the wave front when the amplitude is locally increased. ${ }^{15}$ The geometric result will serve as a reference to which numerical results can be compared.

Five different initial wave forms were created based on particular values for $\mathcal{C}$ and $\mathcal{P}$, most of which are within a range that is plausible for sonic booms. These are grouped 
into two sets of three wave forms; in each set, one parameter is constant while the other is varied.

In the first set, the $(\mathcal{C}, \mathcal{P})$ pairs are $(0.025,0.0001)$, $(0.025,0.0005)$, and $(0.025,0.0025)$. Here, shock amplitude (nonlinearity) is increasing while the shape of the wave front remains the same. For the second set, the values are $(0.0125$, $0.0005),(0.025,0.0005)$, and $(0.05,0.0005)$. In this case, shock amplitude is constant as the initial wave front curvature increases (focal distance decreases). Note that the middle pair of values in each set is the same.

The initial pressure along the $x$ axis at each $y$ value was specified according to the hyperbolic tangent function, Eq. (2). The midpoint of the shock (where $p=0.5 P_{\mathrm{sh}}$ ) lies at $x=-c_{0} \tau_{0}(y)$. The delay time, $\tau_{0}(y)$, is given by Eq. (5). The profile and the wave front, along with the variables that make up $\mathcal{P}$ and $\mathcal{C}$, are illustrated in Fig. 1.

The step size in the $x$ direction, $\Delta x$, is chosen so that the steepest portion of the rise phase of the shock (from 10\% to $90 \%$ of $P_{\mathrm{sh}}$, a distance approximately equal to $l_{\mathrm{sh}}$ ) is resolved by three grid points. The length of the computational domain in the $x$ direction, $L_{x}$, is approximately four times the ripple depth, $L_{0}$. These dimensions require that the number of grid points in the $x$ direction be at least $10 / \mathcal{T}$.

The aspect ratio $\Delta y / \Delta x$ is restricted by the largest angle the wave front makes with respect to the $y$ axis. To avoid an exaggerated staircase shape to the discretized wave front, the aspect ratio is made no larger than $\mathcal{C}^{-1 / 2}$.

The full wave field solution is rendered as a surface plot of acoustic pressure; the positive $x$ axis points to the right (the direction of propagation). In most cases, the solution was carried out to five times the focal distance, $R_{0}$. The results for the first set, in which shock amplitude is varied, are shown in Fig. 3; the weakest shock is shown in plot (a), the strongest shock in plot (c).

The wave field in which nonlinearity is weakest clearly shows the folded wave front pattern predicted by geometrical acoustics; compare the plan view of Fig. 3(a) with the last wave front shown in Fig. 2. Specifically, there is a region along the transverse axis where an observer experiences three distinct shocks. At the edges of this region, the second and third shocks merge into one, where ray theory predicts a caustic. In Fig. 3(a), a secondary shock front can be seen extending beyond the caustics, its amplitude decaying with distance from the caustic. This secondary shock, not predicted by ray theory, is seen in the analytical solution of Obermeier ${ }^{16}$ and in the experimental results of Sturtevant and Kulkarny, ${ }^{14}$ where they are clearly associated with shock fronts diffracting from the sharp edges of the parabolic reflecting surface. In the present context, the secondary shock is believed to be composed of waves diffracted from regions of the initial shock front where the wave front curvature changes from convex to concave.

The evolution of the weakest shock along the central axis is shown in Fig. 4(a). Beyond the point of first focus (where the shock amplitude is largest), the double shock is apparent. The first shock is formed by the intersection of upper and lower parts of the original shock front; rays are crossing here, but not focusing. The second shock is the terminus of rays that have gone through a focus. Over time,

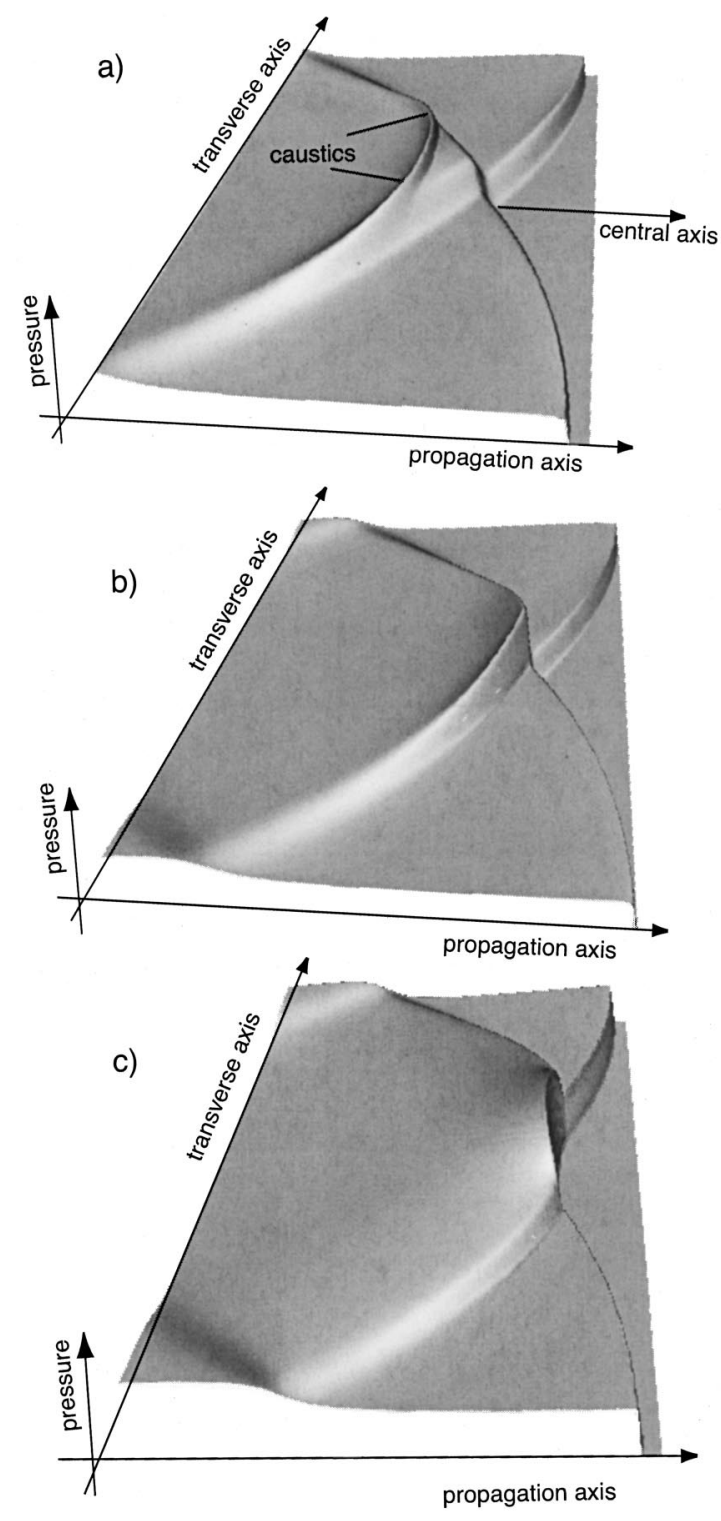

FIG. 3. Behavior of focusing step shock: dependence on shock strength. Shown are full wave field solutions at $t=5 t_{f}$ for three initial conditions: (a) small initial shock amplitude (12 Pa); (b) moderate initial shock amplitude (60 Pa); (c) large initial shock amplitude (300 Pa). Wave front curvature is the same in each case.

the first shock advances relative to the second shock, which is a purely geometric phenomenon.

Note that the amplitude of the second shock decays more rapidly than that of the first shock; this is partly due to the more rapid geometric spreading of the central rays which make up the second shock, but is mostly the result of the nonlinear decay resulting from the rapid pressure decrease behind the second shock. Note also that a slight peak develops on the first shock, similar to what the main shock experiences at the outset. Diffraction from neighboring regions of the wave front (outer diffraction) destructively interferes with the field just behind both shocks on the axis of focus.

By contrast, the wave with the strongest shock amplitude, shown in Fig. 3(c), possesses a wave front that is still smooth and without caustics, consistent with the predictions of shock dynamics. In the vicinity of the central axis there is only one shock, referred to by Obermeier as the "shock 

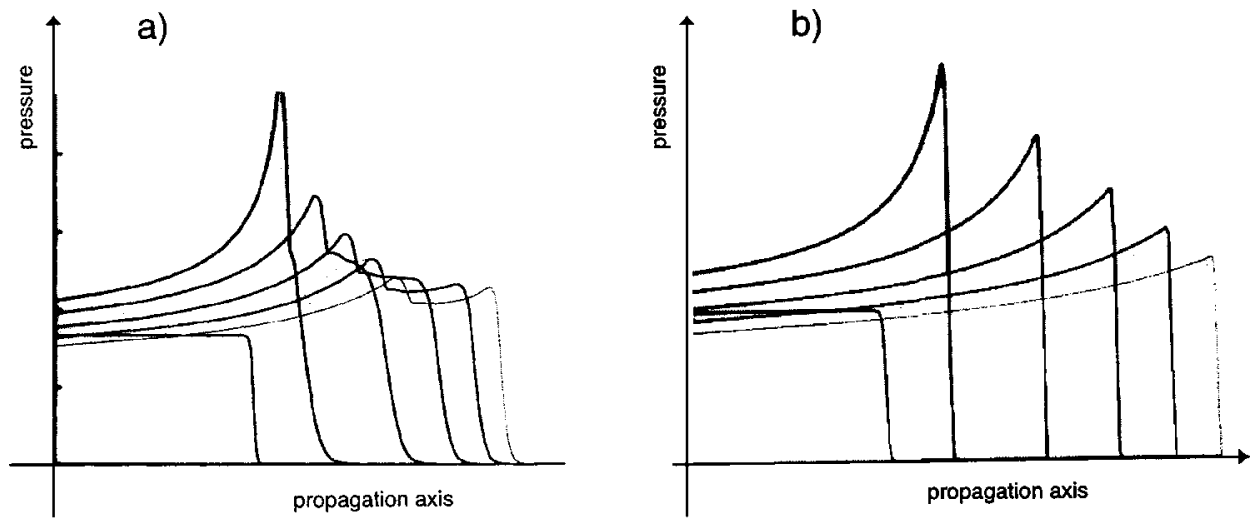

FIG. 4. Evolution of shock profiles along the axis of focus. (a) $\mathcal{C}=.025$, $\mathcal{P}=.0001$; shock front folds after the initial focus at $t=t_{f}$. (b) $\mathcal{C}=.025, \mathcal{P}$ $=.025 ;$ shock front does not fold. The initial amplitude in (b) is 25 times larger than in (a). Profiles are plotted at $t=0, t=t_{f}, \quad t=2 t_{f}, \quad t=3 t_{f}, \quad t$ $=4 t_{f}$, and $t=5 t_{f}$. disk." Outside of this shock disk a second shock front is observed whose amplitude decays with distance from the central axis. This feature is also seen in the solution of Obermeier $^{16}$ and in the photographs of Sturtevant and Kulkarney. ${ }^{14}$ At the top and bottom corners on the left-hand side of the plot can be seen wave fronts reflecting off the upper and lower boundaries.

The evolution of the wave form along the central axis is shown in Fig. 4(b). Note that the vertical scale is not the same as in plot (a) of the same figure; the initial shock amplitudes in the two cases differ by a factor of 25 . With no folding, the pressure profile contains only a single shock, followed by a logarithmically decreasing overpressure. The shock amplitude decreases in accordance with the predictions of shock dynamics.

The shock front in Fig. 4(b) advances relative to the reference frame of the computational domain with speed $v_{\text {sh }}-c_{0}=0.5 c_{0} \mathcal{P}$, which is proportional to the shock amplitude. Thus, the shock speed is greatest along the central axis and decreases with distance from this axis, causing the wave front curvature to decrease with time.

The solution shown in Fig. 3(b) appears to represent a middle ground between geometric acoustics and shock dynamics; the wave front is neither folded, nor does it clearly show self-refraction. With this result as a reference $(\mathcal{P}$ $=0.0005$ and $\mathcal{C}=0.025$ ), two further numerical trials were performed in which the initial wave front curvature was changed (shock amplitude is held constant). When $\mathcal{C}$ is increased from 0.025 to 0.05 (corresponding to a decrease in the focal distance, $R_{0}$ ), the wave exhibits geometric folding behavior, as shown in Fig. 5(a). When $\mathcal{C}=0.0125$, corresponding to a more shallow curvature, the wave is clearly in the shock dynamic regime, as shown in Fig. 5(b).

These numerical results confirm the presence of a transition between geometric and shock dynamic behavior for focusing shocks that have amplitudes and curvatures representative of sonic booms. References 16 and 14 show this transition for shocks with larger amplitudes and shorter focus lengths. Also demonstrated is the efficacy of describing a curved shock front with two nondimensional parameters, each quantifying the role played by nonlinearity or diffraction. ${ }^{17}$

\section{SINUSOIDAL WAVE FRONT RIPPLING}

\section{A. Connection to atmospheric inhomogeneities}

A simple model of atmospheric turbulence, adapted from Panofsky and Dutton, ${ }^{18}$ describes turbulence as a collection of vortices. Each vortex is specified by a characteristic length (diameter), $L_{t}$, and a characteristic tangential
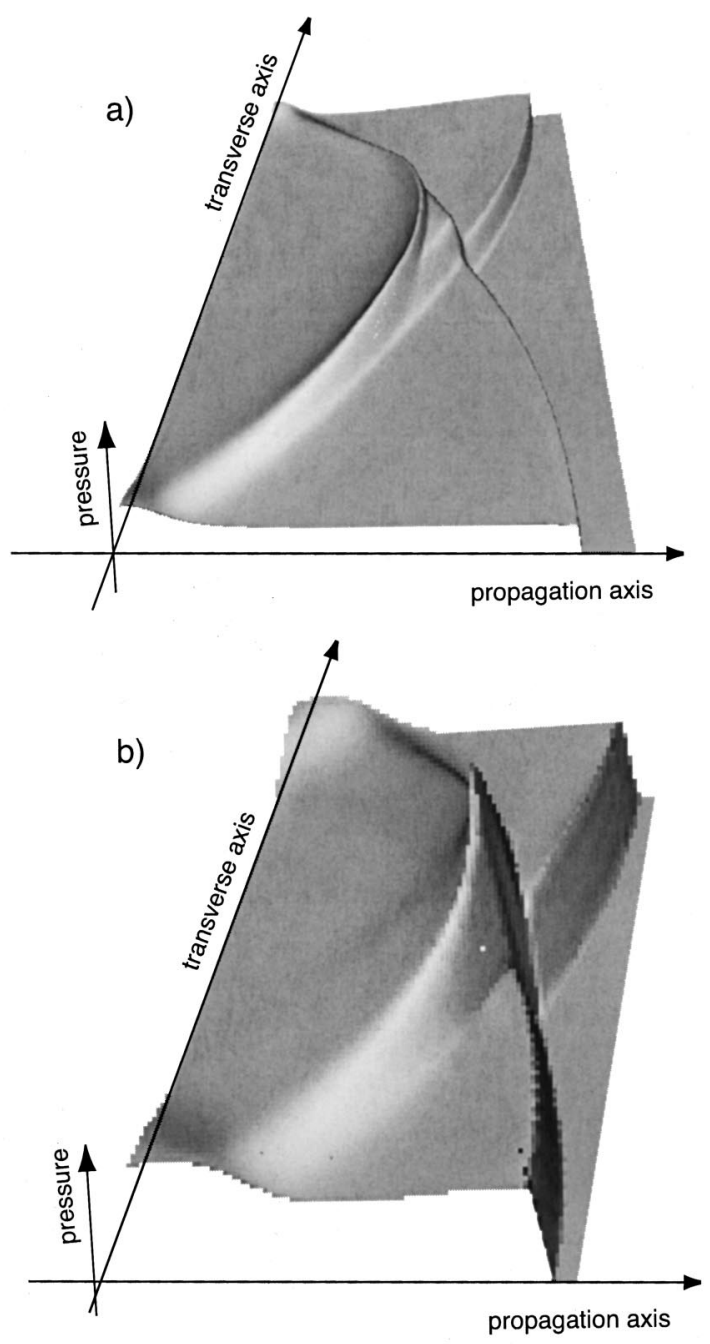

FIG. 5. Behavior of focusing step shock: dependence on wave front curvature. Shown are full wave field solutions at $t=5 t_{f}$ : (a) large initial curvature (short focal length); (b) small initial curvature (large focal length). 

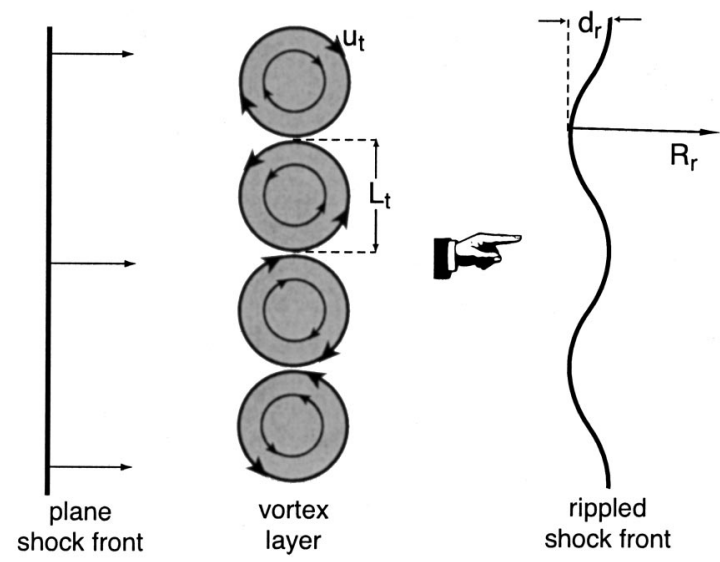

FIG. 6. Illustration depicting the rippling of an initially plane shock front due to a chain of vortices. Ripple parameters $R_{r}$ and $d_{r}$ can be expressed in terms of vortex parameters $L_{t}$ and $u_{t}$.

speed, $u_{t}$, at the outer edge. Within this framework, one of the simplest realizations of turbulence is a thin layer consisting of a linear chain of identical vortices alternating in their direction of rotation, like a series of gears. An initially plane wave front passing through this vortex layer will become rippled in a way that is approximately sinusoidal with wave number $k_{r}=\pi / L_{t}$. This scenario is illustrated in Fig. 6 .

The amplitude, $d_{r}$, and the minimum radius of curvature, $R_{r}$, of a sinusoidal ripple can be expressed (to first order) in terms of the vortex parameters as follows:

$$
\begin{aligned}
& d_{r}=\frac{L_{t} u_{t}}{c}, \\
& R_{r}=\frac{L_{t} c}{10 u_{t}} .
\end{aligned}
$$

Note that $d_{r}$ corresponds to $L_{0}$ of the parabolic ripple, described in Eq. (5). The nondimensional parameter associated with wave front curvature is then $\mathcal{C}=d_{r} / R_{r}$ $=10\left(u_{t} / c\right)^{2}$. Thus, even if $u_{t}$ and $L_{t}$ both independently characterize vortices, the focusing behavior of a rippled wave front, relative to the shock amplitude, depends only on $u_{t}$. This is physically plausible, since increasing the size of the vortex (while maintaining a constant outer velocity) will both increase the amplitude of the ripple and decrease the ripple wave number, with the net result that the ripple curvature is approximately constant.

The vortex layer just described can be interpreted as a realization of a single wave number component of turbulence. A more complete description of turbulence can be obtained via the superposition of many vortex streets within a thin layer, each representing a different component of turbulence. Wave front rippling from such a superposition of vortices will be a linear superposition of sinusoidal ripples that would result from each component alone. In this way, the effects of turbulence within a thin layer can be modeled directly with wave front rippling.

It should be emphasized that the rippling occurs along only one axis parallel to the wave front; the wave front is uniform along the other axis. The other important simplifi- a)
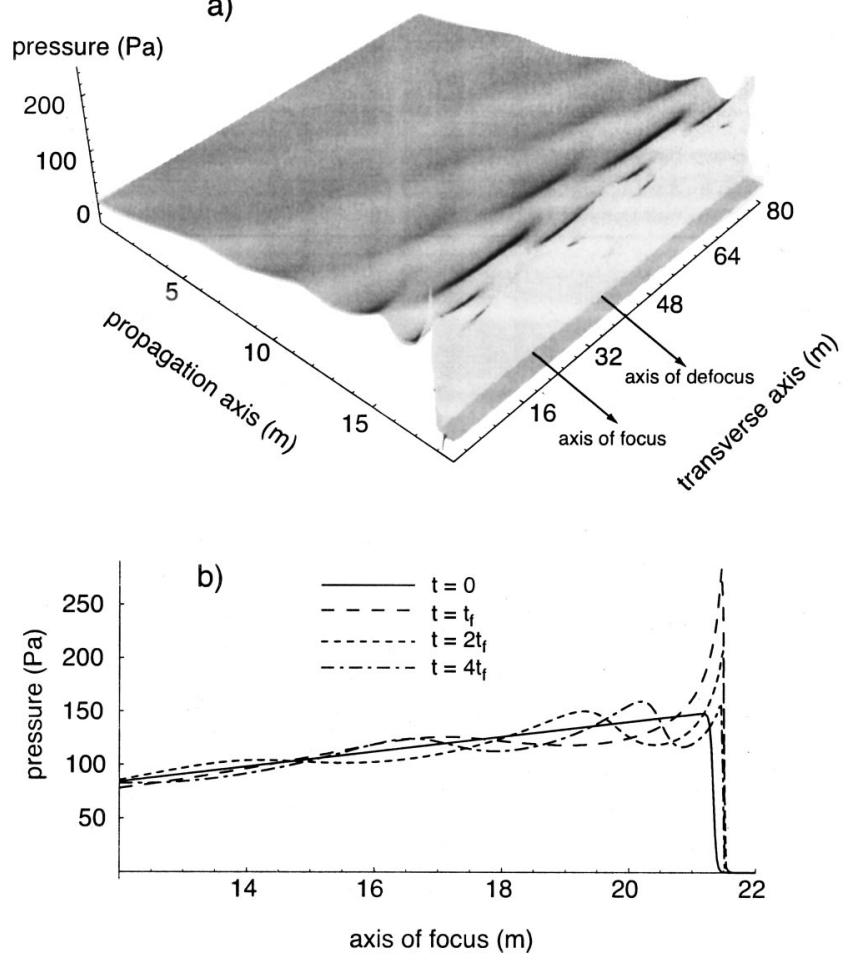

FIG. 7. Solution for positive phase of sonic boom with shallow sinusoidal ripple: case I. (a) Full pressure field at $t=2.4 \mathrm{~s}$, (b) evolution of shock profile along axis of focus.

cation is that the effects of atmospheric inhomogeneities are realized only for the initial condition. The numerical solution assumes a homogeneous medium.

Section III B describes the numerical experiment that was conducted to assess the contributions to shock profile distortion from different scales of wave front rippling.

\section{B. Description of the numerical experiment}

The initial pressure field consists of the positive phase of an $N$ wave with a sinusoidally rippled wave front. The wave form has approximately the shape of a right triangle: at the leading edge, the shock is described by the Taylor profile [Eq. (2)]; behind the shock, the overpressure decreases linearly to zero. The length of the pulse is $20 \mathrm{~m}$, derived from a typical sonic boom duration of $120 \mathrm{~ms}$.

Three cases, corresponding to different initial conditions, were studied. In cases I and II, the wave front is sinusoidally rippled from a single chain of vortices; in case III, the ripple has two wave number components. In each case the shock amplitude is $150 \mathrm{~Pa}(\mathcal{P}=0.0013)$, resulting in a shock width of $0.1 \mathrm{~m}$.

The first two cases have different initial wave front curvature. In case I, vortices with diameter $L_{t}=20 \mathrm{~m}$ and speed $u_{t}=5 \mathrm{~m} / \mathrm{s}$ produce a ripple depth $d_{r}=0.3 \mathrm{~m}$ and focal distance $R_{r}=128 \mathrm{~m}$; then $\mathcal{C}=0.0024$ and $\mathcal{T}=0.33$. In case II, vortices have diameter $L_{t}=40 \mathrm{~m}$ and speed $u_{t}=12 \mathrm{~m} / \mathrm{s}$, so that $d_{r}=1.4 \mathrm{~m}$ and $R_{r}=112 \mathrm{~m}$; the resulting curvature is $\mathcal{C}=0.0125$ and the thickness is $\mathcal{T}=0.071$.

In case III, the wave front ripple has two components, described by parameters $\mathcal{C}_{1}=0.0125$ and $\mathcal{C}_{2}=0.0015$. This wave front was generated by adding a second component to 
a)

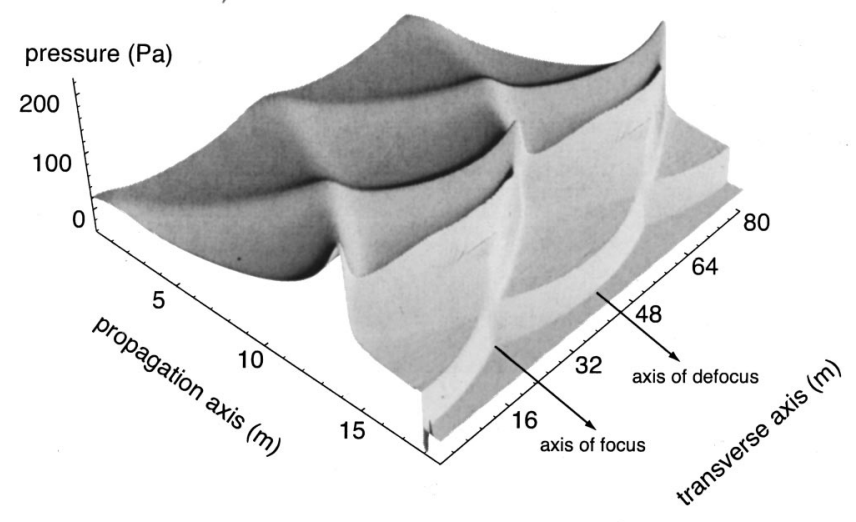

b)

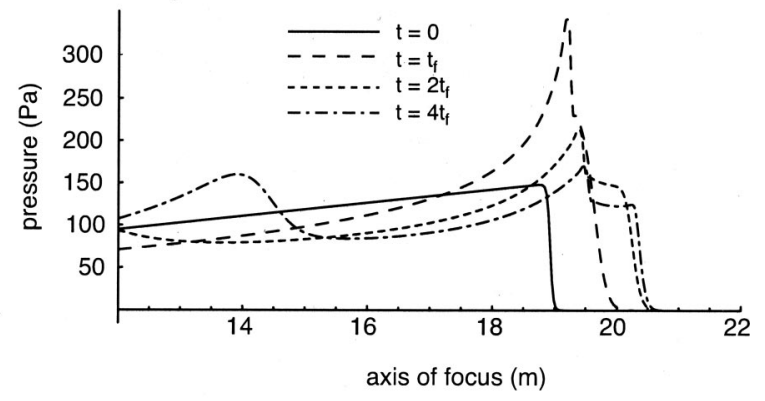

FIG. 8. Solution for positive phase of sonic boom with deep sinusoidal ripple: case II. (a) Full pressure field at $t=1.6 \mathrm{~s}$, (b) evolution of shock profile along axis of focus.

the rippling of case II. The new component has a higher wave number, which is produced by smaller $\left(L_{t}=8 \mathrm{~m}\right)$ and slower $\left(u_{t}=4 \mathrm{~m} / \mathrm{s}\right)$ vortices; most importantly (with regard to ripple curvature), the ripple depth of the second component is smaller: $d_{r}=0.1 \mathrm{~m}$. An interesting feature of the higher wave number ripple component is that the ripple depth equals the shock thickness $(\mathcal{T}=1)$.

A final comment about the initial conditions should be made regarding the use of $\mathcal{C}$ to predict focusing behavior. In all three of the sinusoidal ripple cases $\mathcal{C}$ is equal to, or smaller than, the smallest value used in the step shocks with a parabolic ripple $(\mathcal{C}=0.0125)$. In the latter case, the shock did not fold. Since the amplitude of the sinusoidally rippled $N$ waves is also larger, one might expect that they should all behave according to shock dynamics, if the parameter $\mathcal{C}$ may be meaningfully compared among different curvature shapes. The results suggest otherwise.

\section{RESULTS}

\section{A. Single ripple}

Numerical results for case I (small wave front curvature) are summarized in Fig. 7 and the results for case II (large curvature) are shown in Fig. 8. Plot (a) in Figs. 7 and 8 shows the full wave field at a propagation distance well beyond the point of first focus; plot (b) shows the time evolution of the shock profile along the axis of focus indicated in plot (a).

These plots clearly show that the shock front in case I does not fold (it is nearly planar), while that of case II does
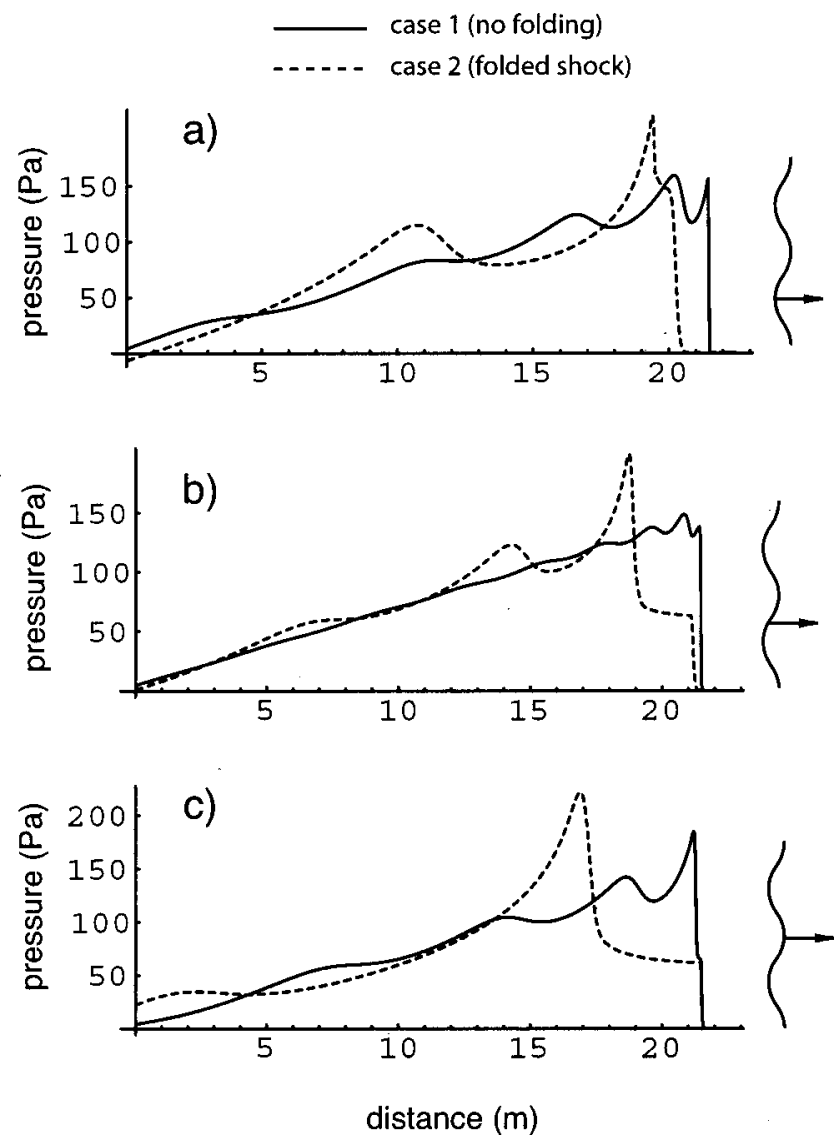

FIG. 9. Shock profiles of two sinusoidally rippled $N$ waves that have propagated beyond the point of initial focus; one shock front has experienced folding (dashed curve), the other has not (solid curve). (a) Shock profiles recorded on axis of focus; (b) shock profiles recorded on the axis that intersects an inflection point on the initial wave front ripple; (c) shock profiles recorded on an axis of defocus.

fold. It is also evident that the peak amplitude on the axis of focus is larger in the folding case, where it occurs at the second shock (behind the leading shock). When the wave front does not fold, there is only one shock. These results confirm the shock dynamics prediction that strong nonlinear effects contribute to a decrease in the shock amplitude. ${ }^{15}$

The periodic form of the rippling produces a sort of interference (or "waffle") pattern behind the shock front in both cases. This corresponds to humps seen in the shock profiles. As propagation continues, the humps steepen and progress toward the shock front. In the case of no folding, the amplitude of the first hump eventually exceeds that of the shock [see Fig. 7(b)].

The observed pressure wave form depends on the location of the observer relative to the rippling in the wave front. The profiles in Figs. 7 and 8 show what would be measured by an observer situated along the axis of focus. An observer located at some other point on the transverse axis would measure a different wave form.

Figure 9 shows pressure profiles for both cases at three different observer locations along the transverse axis: the axis of focus, the axis of defocus, and a point midway between these. The folding shock exhibits significant variation along the transverse axis. Away from the axis of focus, the initial shock decreases in amplitude and advances relative to 
the larger second shock. By contrast, the shock front that does not fold thickens only slightly away from the axis of focus. At all three observation points, the folded shock exhibits the larger amplitude spike.

As with the parabolic wave front curvature, there must exist some shocks with sinusoidal rippling that exhibit neither definite geometric nor definite shock dynamic focusing behavior. Since the numerical results described previously correspond to initial conditions that are well within the realm of possible rippling produced by actual atmospheric turbulence, one may conclude that not all sonic booms will experience folding (at least if rippling shapes are approximately parabolic or sinusoidal).

\section{B. Multiple ripples}

The additional ripple component in case III is an independent source of spikes and other wave form distortions due to focusing, although the combined effects do not arise from a linear process of superposition. The initial wave front curvature of the additional ripple component is small enough $(\mathcal{C}=0.0015)$ that it does not lead to wave front folding, unlike the larger scale ripple. Between the two ripple components there is zero phase difference at the axes of focus and defocus; both ripple components are focusing and defocusing together along these axes, albeit at different rates.

Results for case III can be compared with the singlecomponent case II in Fig. 10. The pressure profiles of both cases are plotted together at three observer locations, with the dashed curved representing case III. The profiles all come from the full-field solution at $t=1.6 \mathrm{~s}$, at which point the shock has propagated approximately five times farther than the focal distance, $R_{r}$. The case III profiles, particularly along the axes of focus and defocus, exhibit small peaks in the vicinity of the shock front not seen in the corresponding case II profiles. Behind the main shock in each case, little difference is seen between the two cases. This is to be expected, because the smaller length scales $\left(L_{t}\right.$ and $\left.d_{r}\right)$ of the extra ripple component correspond to a smaller domain of influence in the field behind the shock front.

On the axis of focus, the case III profile exhibits a peak at the leading shock [Fig. 10(a)], compared to the smooth step bridging the first and second shocks seen in case II. This may be explained as the approximate superposition of the case II wave field with a nonfolding wave field similar to that seen in plot (a) of Fig. 7.

A somewhat more surprising result is the slight rounding (or delayed maximum) of the initial shock seen in the profile on the axis of defocus [Fig. 10(c)]. Simple superposition of folding and nonfolding wave fields does not satisfactorily explain the observed result.

One feature of these ripples that turned out to be less significant than anticipated is the shock thickness parameter, $\mathcal{T}=l_{\mathrm{sh}} / d_{r}$. When $\mathcal{T} \ll 1$, the shock is abrupt (as perceived by an observer sufficiently remote from the wave front to see that it contains many ripples); the more abrupt the shock, the better geometric theory (linear or nonlinear) will describe the evolution of the shock front.

If $\mathcal{T}$ is close to, or greater than, unity, as is true for the larger ripple wave number component in case III, the shock
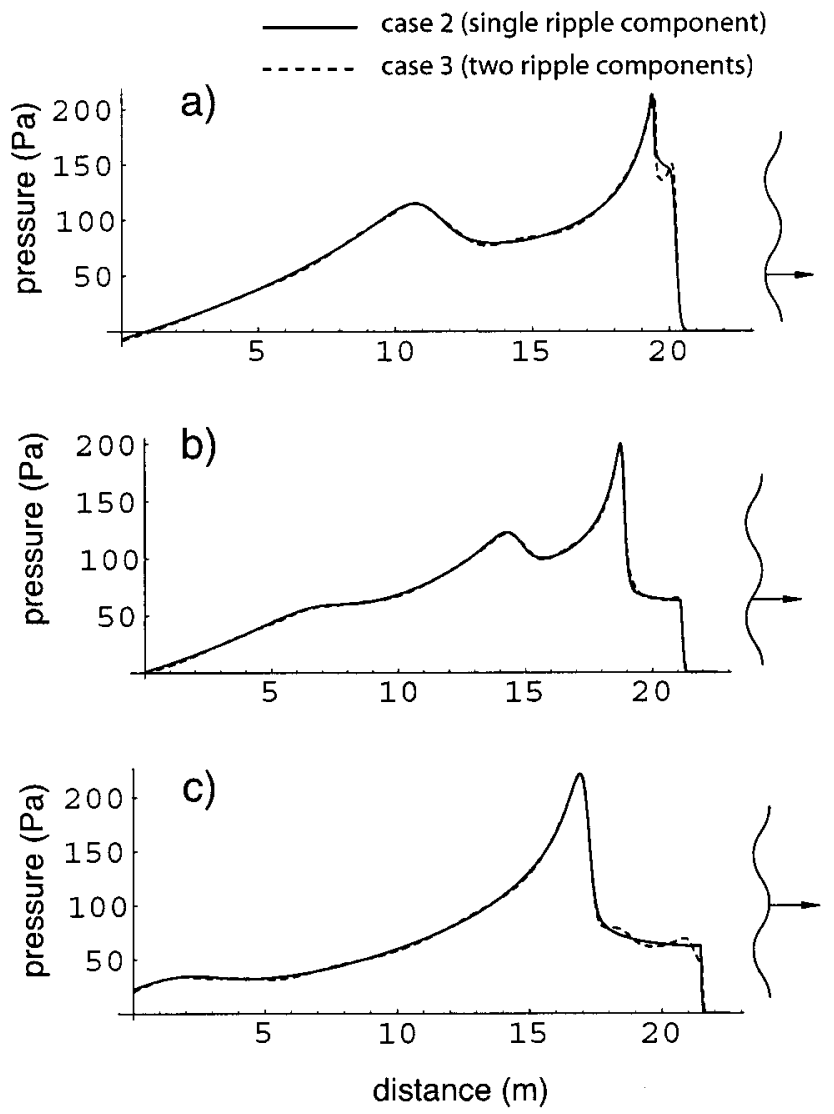

FIG. 10. Shock profiles of rippled $N$ waves that have propagated beyond the point of initial focus; one shock front is rippled with a single sinusoid component (solid line), the other is rippled with two sinusoid components (dashed line). (a) Shock profiles recorded on axis of focus; (b) shock profiles recorded on the axis that intersects an inflection point on the initial wave front ripple; (c) shock profiles recorded on an axis of defocus.

has a curvature comparable to its rise phase and would thus be far from the geometric approximation. In this case, it might be expected that, as focusing occurs, acoustic energy is readily diffracted away from ray paths, so that local increases in pressure are less pronounced.

The results demonstrate, however, that focusing of shallow ripples still produces distinct peaks at (and near) the shock front. An important implication is that even weak sonic booms (amplitudes less than $100 \mathrm{~Pa}$ ), which are relatively thick due to molecular relaxation, will exhibit spiky features due to focusing.

\section{CONCLUSIONS}

A numerical study of the propagation of sonic booms with rippled wave fronts was performed in order to qualitatively and, to some extent, quantitatively evaluate the kind of wave form distortions that might be produced by various scales of wave front rippling. Via a simple model, rippling scales are associated with atmospheric turbulence parameters.

Whether folding of sonic boom wave fronts occurs depends on the amount of curvature present in the turbulence induced wave front rippling. Numerical results indicate that even for a relatively large amplitude $(150 \mathrm{~Pa})$, rippling produced by plausible turbulence conditions will result in a 
folded wave front. For a given wave front curvature, weaker shocks have a greater tendency to fold, implying that for weaker sonic booms, only the less energetic components of turbulence will produce ripples that do not lead to folding.

The wave form distortions associated with geometric wave front folding are distinctly different from those associated with shock dynamic wave front straightening. In both cases, continuous rippling along the wave front produces humps in the wave form behind the shock that advance and steepen. Folded shocks, however, exhibit a step between a leading shock and a stronger main shock; the time delay between these two shocks and the difference in their amplitudes increases significantly as an observer moves from the axis of focus to an axis of defocus.

Another discernible difference between folded and nonfolded shocks, regardless of observer location, is that folded shocks have large, distinct spikes (associated with the main shock), whereas nonfolded shocks have smaller spikes clustered near the shock front. This appears to be true for propagation distances between two and eight times the focal distance (the latter is typically between 50 and $100 \mathrm{~m}$ for sinusoidal ripples).

Finally, multiple ripple components independently appear to produce wave form distortions that are appropriate to their respective scales; at moderate propagation distances (between five and ten focal lengths), the combined effect on the pressure signature is approximately the superposition of these distinct processes. In particular, small peaks near the shock front are seen to be superimposed on the larger scale features when a second, smaller ripple component is added to the initial wave front.

Although the computational experiment simulated a very simple scenario, the particular outcomes contain many of the features seen in actual sonic boom recordings. ${ }^{2}$ Among these are small spikes near the shock, large spikes far behind the shock front, and moderate rounding of the shock. One may also tentatively conclude that distortions due to small-scale focusing contribute high-frequency energy in the shock within moderate propagation distances beyond the initial rippling; as propagation continues, the smaller peaks near the shock front are steadily eroded by nonlinear steepening.

The present results are sufficiently encouraging to warrant pursuing more sophisticated numerical modeling of rippled wave fronts. Instead of constructing wave fronts from Fourier components, turbulence could be directly incorporated into the propagation scheme via velocity perturbations at each grid point. To make the problem computationally feasible, the sonic boom should be discretized on a nonuniform mesh, such that the very fine resolution is applied only near the shock front.

\section{ACKNOWLEDGMENTS}

The author wishes to acknowledge the scientific support and critical feedback, especially from John White and Doug Clarke, that he received while working on this project as a postdoctoral fellow at the Lawrence Livermore National Laboratory.

${ }^{1}$ A. Niedzwiecki and H. S. Ribner, J. Acoust. Soc. Am. 64, 1622-1626 (1978).

${ }^{2}$ D. J. Maglieri, V. Huckel, H. R. Henderson, and N. J. McLeod, "Variability in sonic-boom signatures measured along an 8000-foot linear array," NASA TN D-5040 (1969).

${ }^{3}$ S. C. Crow, "Distortion of sonic bangs by atmospheric turbulence," J. Fluid Mech. 37, 529-563 (1969).

${ }^{4}$ A. D. Pierce, "Spikes on sonic-boom pressure wave forms," J. Acoust. Soc. Am. 44, 1052-1061 (1968).

${ }^{5}$ A. D. Pierce and D. J. Maglieri, "Effects of atmospheric irregularities on sonic-boom propagation," J. Acoust. Soc. Am. 51, 7022-721 (1972).

${ }^{6}$ A. D. Pierce, "Statistical theory of atmospheric turbulence effects on sonic-boom rise times," J. Acoust. Soc. Am. 49, 906-924 (1971).

${ }^{7}$ K. J. Plotkin and A. R. George, "Propagation of weak shock waves through turbulence,"' J. Fluid Mech. 54, 449-467 (1972).

${ }^{8}$ K. J. Plotkin, "The effect of turbulence on the loudness of minimized sonic boom signatures," NASA Conference on High-speed Research (1992).

${ }^{9}$ B. E. McDonald and W. A. Kuperman, "Time domain formulation for pulse propagation including nonlinear behavior at a caustic,', J. Acoust. Soc. Am. 81, 1406-1417 (1987).

${ }^{10}$ A. A. Piacsek, "A numerical study of weak step shocks that focus in two dimensions," Ph.D. thesis, Pennsylvania State University, 1995.

${ }^{11}$ B. E. McDonald and J. Ambrosiano, "High-order upwind flux correction methods for hyperbolic conservation laws,' J. Comput. Phys. 56, 448460 (1984).

${ }^{12}$ J. P. Hodgson, "Vibrational relaxation effects in weak shock waves in air and the structure of sonic bangs," J. Fluid Mech. 58, 187-196 (1973).

${ }^{13}$ J. Kang, "Nonlinear acoustic propagation of shock waves through the atmosphere with molecular relaxation," Ph.D. thesis, Pennsylvania State University, 1991.

${ }^{14}$ B. Sturtevant and V. A. Kulkarny, "The focusing of weak shock waves," J. Fluid Mech. 73, 651-671 (1976).

${ }^{15}$ G. B. Whitham, "On the propagation of weak shock waves," J. Fluid Mech. 1, 290-318 (1956).

${ }^{16} \mathrm{~F}$. Obermeier, "'On the propagation of weak and moderately strong curved shock waves," J. Fluid Mech. 129, 123-136 (1983).

${ }^{17}$ W. L. Willshire, Jr. and D. W. Devilbiss, "Preliminary results from the White Sands Missile Range sonic boom propagation experiment" NASA Conference on High-speed Research, (1992), pp. 137-149.

${ }^{18} \mathrm{H}$. Panofsky and J. Dutton, Atmospheric Turbulence: Models and Methods for Engineering Applications (Wiley, New York, 1984), p. 35. 